\title{
Beitrag zur Theorie der linearen partiellen Differentialgleichungen.
}

\author{
(Von Herrn R. Lipschitz in Bonn.)
}

Verschiedene physikalische Untersuchungen führen auf analytische Aufgaben, die in der folgenden Forderung enthalten sind. Wenn ein Ort im Raume durch die rechtwinkligen Coordinaten $x, y, z$ bestimmt ist, und wenn für das Innere eines gewissen endlichen Raumes $T$ die eindeutigen, endlichen und stetigen Functionen des Ortes $u, v, w, g$ gegeben sind, so sollen vier Functionen $\boldsymbol{P}, \boldsymbol{P}_{23}, \boldsymbol{P}_{31}, \boldsymbol{P}_{12}$ gefunden werden, die den vier Gleichungen

$$
\begin{aligned}
& u=\frac{\partial P}{\partial x}+\frac{\partial P_{12}}{\partial y}-\frac{\partial P_{31}}{\partial z} \\
& v=-\frac{\partial P_{12}}{\partial x}+\frac{\partial P}{\partial y}+\frac{\partial P_{23}}{\partial z} \\
& w=\frac{\partial P_{31}}{\partial x}-\frac{\partial P_{23}}{\partial y}+\frac{\partial P}{\partial z} \\
& g=\frac{\partial P_{23}}{\partial x}+\frac{\partial P_{31}}{\partial y}+\frac{\partial P_{12}}{\partial z}
\end{aligned}
$$

genügen. Herr Helmholtz hat in dem Aufsatze ,über Integrale der hydrodynamischen Gleichungen, welche den Wirbelbewegungen entsprechen ${ }^{6}$ (Bd. 55 dieses Journals, p. 25) diese Aufgabe gestellt und aufgelöst, indem er voraussetzt, dass für jeden Punkt von $T$ die Function $g$ gleich der Null sei, und die Functionen $u, v, w$ die Gleichung

$$
\frac{\partial u}{\partial x}+\frac{\partial v}{\partial y}+\frac{\partial w}{\partial z}=\mathbf{0}
$$

befriedigen, dass ferner für jeden Punkt der den Raum $T$ begrenzenden 0berfläche $S$, wenn die von dem Raume $T$ nach aussen gezogene Flächennormale $n$ mit den positiven Axen der $x, y, z$ beziehungsweise die Winkel $\alpha, \beta, \gamma$ bildet, die Gleichung

$$
\left(\frac{\partial v}{\partial z}-\frac{\partial w}{\partial y}\right) \cos \alpha+\left(\frac{\partial w}{\partial x}-\frac{\partial u}{\partial z}\right) \cos \beta+\left(\frac{\partial u}{\partial y}-\frac{\partial v}{\partial x}\right) \cos \gamma=0
$$

erfüllt sei. Mit der alleinigen Einschränkung, dass die Function $g$ überall gleich der Null sei, ist das obige Problem von Roch in seiner Dissertation „Anwendung der Potentialausdrücke auf die Theorie der molecularphysikalischen 
110 Lipschitz, Beitrag zur Theorie der linearen partiellen Differentialgleichungen.

Fernwirkungen etc. ${ }^{66}$ (Bd. 61 dieses Journals, p. 283) behandelt worden. Daselbst wird bemerkt, dass jede der Functionen $P, P_{23}, P_{31}, P_{12}$ als das $\mathrm{Ag}-$ gregat von zwei Integralen ausgedrückt werden könne, von denen das eine über den Raum $T$, das andere über die Oberfläche $S$ auszudehnen ist. Zugleich erfolgt eine analytische Bestimmung der betreffenden Raumintegrale und eine Deutung derselben, die sich nach dem Vorgange von Herrn Helmholtz auf die Analogie zu dem Wirkungsgesetze der ruhenden und der bewegten electrischen Massen stützt.

Die allgemeinere Fassung, die dem Problem oben gegeben ist, wird durch die Symmetrie der gegenwärtig zu entwickelnden Auflösung, wie ich glaube, gerechtfertigt erscheinen. Die gefundenen Werthe der Functionen $P, P_{23}, P_{31}, P_{12}$ treten in ihrer ersten Gestalt als Integrale auf, die sich uber den Raum $T$ erstrecken. Dadurch, dass jedes dieser Integrale in das Aggregat eines Raumintegrals und eines Oberflächenintegrals aufgelöst wird, entsteht eine zweite Gestalt der Auflösung. Die erste Gestalt bietet einen Gesichtspunkt zu einer physikalischen Deutung der vollständigen gefundenen Werthe der Functionen $P, P_{23}, P_{31}, P_{12}$. Die zweite Gestalt liefert die Form der Raumintegrale, welche in dem angeführten Rochschen Aufsatze unter der dort geltenden Voraussetzung angegeben und gedeutet ist, und die Form der Oberflächenintegrale, welche daselbst nicht bestimmt ist, aber in entsprechender Weise gedeutet werden kann.

\section{1.}

Vor Erörterung des Problems wird es angemessen sein zu erwägen, welchen Charakter die allgemeinste Auflösung desselben habe. Gesetzt es seien $P=P, P_{23}=P_{23}, P_{31}=P_{31}, P_{12}=P_{12}$ und $P=Q, P_{23}=Q_{23}, P_{31}=Q_{31}$, $P_{12}=Q_{12}$ zwei Systeme von Auflösungen, so befriedigen die Differenzen $Q-P=\sigma, Q_{23}-P_{23}=\sigma_{23}, Q_{31}-P_{31}=\sigma_{31}, Q_{12}-P_{12}=\sigma_{12}$ das von den Werthen der gegebenen Functionen $u, v, w, g$ unabhängige System von Gleichungen

$$
\begin{aligned}
& 0=\frac{\partial \sigma}{\partial x}+\frac{\partial \sigma_{12}}{\partial y}-\frac{\partial \sigma_{31}}{\partial z}, \\
& 0=-\frac{\partial \sigma_{12}}{\partial x}+\frac{\partial \sigma}{\partial y}+\frac{\partial \sigma_{23}}{\partial z}, \\
& 0=\frac{\partial \sigma_{31}^{\circ}}{\partial x}-\frac{\partial \sigma_{23}}{\partial y}+\frac{\partial \sigma}{\partial z}, \\
& 0=\frac{\partial \sigma_{23}}{\partial x}+\frac{\partial \sigma_{31}}{\partial y}+\frac{\partial \sigma_{12}}{\partial z},
\end{aligned}
$$


Lipschitz, Beitrag zur Theorie der linearen partiellen Differentialgleichungen. 111

welches bei Anwendung der Charakteristik $\Delta f=\frac{\partial^{2} f}{\partial x^{2}}+\frac{\partial^{2} f}{\partial y^{2}}+\frac{\partial^{2} f}{\partial z^{2}}$ die vier Gleichungen $0=\Delta \sigma, 0=\Delta \sigma_{23}, 0=\Delta \sigma_{31}, 0=\Delta \sigma_{12}$ nach sich zieht. Demnach sieht man, dass, wenn ein System von Auflösungen $\boldsymbol{P}, \boldsymbol{P}_{23}, \boldsymbol{P}_{31}, \boldsymbol{P}_{12}$ mit allen Systemen von Auflösungen des vorstehenden Systems von Gleichungen $\sigma, \sigma_{23}$, $\sigma_{31}, \sigma_{12}$, verbunden wird, die Functionen $P+\sigma, P_{23}+\sigma_{23}, P_{31}+\sigma_{31}, P_{12}+\sigma_{12}$ die sämmtlichen Systeme von Auflösungen unserer Aufgabe und zwar jedes ein Mal darstellen. Wir werden daher nur ein einziges System von Functionen $\boldsymbol{P}, \boldsymbol{P}_{23}, \boldsymbol{P}_{31}, \boldsymbol{P}_{12}$ aufsuchen, das unsere Aufgabe erfüllt.

Diese Absicht lässt sich erreichen, indem man annimmt, dass die Functionen $P, P_{23}, P_{31}, P_{12}$ von vier neuen Functionen $U, V, W, G$ in der folgenden Weise abhängen

$$
\left\{\begin{array}{l}
\boldsymbol{P}_{23}=\frac{\partial \boldsymbol{G}}{\partial \boldsymbol{x}}-\frac{\partial \boldsymbol{W}}{\partial y}+\frac{\partial \boldsymbol{V}}{\partial z} \\
\boldsymbol{P}_{31}=\frac{\partial \boldsymbol{W}}{\partial \boldsymbol{x}}+\frac{\partial \boldsymbol{G}}{\partial y}-\frac{\partial \boldsymbol{U}}{\partial z} \\
\boldsymbol{P}_{\mathbf{1 2}}=-\frac{\partial \boldsymbol{V}}{\partial \boldsymbol{x}}+\frac{\partial \boldsymbol{U}}{\partial y}+\frac{\partial \boldsymbol{G}}{\partial z} \\
\boldsymbol{P}=\frac{\partial \boldsymbol{U}}{\partial \boldsymbol{x}}+\frac{\partial \boldsymbol{V}}{\partial y}+\frac{\partial \boldsymbol{W}}{\partial z}
\end{array}\right.
$$

und verlangt, dass die Functionen $U, V, W, G$ der aufgestellten Forderung gemäss bestimmt werden. Die Substitution dieser Ausdrücke in die gegebenen Gleichungen

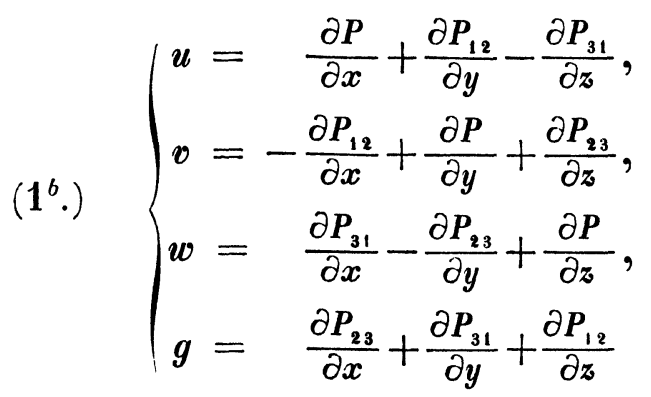

liefert dann die Gleichungen

$$
\text { (2.) } \quad u=\Delta U, \quad v=\Delta V, \quad w=\Delta W, \quad g=\Delta G,
$$

von denen jede zu der Bestimmung von einer der Functionen $U, V, W, G$ geeignet ist. Wenn man festsetzt, dass eine Function $f$ von $x, y, z$ durch die Substitution $x=x^{\prime}, y=y^{\prime}, z=z^{\prime}$ in die Function $f^{\prime}$ übergehe, dass der positive Werth $\sqrt{ }\left(\left(x-x^{\prime}\right)^{2}+\left(y-y^{\prime}\right)^{2}+\left(z-z^{\prime}\right)^{2}\right)=r$ sei, und dass das Volumen- 
element $d x^{\prime} d y^{\prime} d z^{\prime}$ mit $d t^{\prime}$ bezeichnet werde, so erfüllen bekanntlich die über den Raum $T$ auszudehnenden Integrale

$$
\left\{\begin{array}{c}
U=-\frac{1}{4 \pi} \int \frac{u^{\prime} d t^{\prime}}{r}, V=-\frac{1}{4 \pi} \int \frac{v^{\prime} d t^{\prime}}{r}, W=-\frac{1}{4 \pi} \int \frac{w^{\prime} d t^{\prime}}{r}, \\
G=-\frac{1}{4 \pi} \int \frac{g^{\prime} d t^{\prime}}{r}
\end{array}\right.
$$

die Gleichungen (2.).

Da die Functionen $u, v, w, g$ nach der getroffenen Voraussetzung in dem Raume $T$ eindeutig, endlich und stetig sind, so stellen die für $U, V, W, G$ gefundenen Integrale Functionen dar, die mit Einschluss der nach $x, y, z$ genommenen Differentialquotienten erster und zweiter Ordnung überall im Raume $T$ eindeutig und endlich sind. Auch kann man bemerken, dass diese Integrale, wenn $x, y, z$ die Coordinaten eines Punktes bedeuten, der ausserhalb des Raumes $T$ liegt, die partiellen Differentialgleichungen $0=\Delta u, 0=\Delta v, 0=\Delta w$, $0=\Delta g$ befriedigen. Indem man nun die Werthe von $u, v, w, g$ in die Gleichungen $\left(1^{a}\right.$.) substituirt, die Differentiationen nach $x, y, z$ unter die Integralzeichen bringt, und daselbst durch Differentiationen nach $x^{\prime}, y^{\prime}, z^{\prime}$ ersetzt, so entsteht das folgende System von Functionen $P, P_{23}, P_{31}, P_{12}$, welches die gestellte Aufgabe löst,

$$
\left\{\begin{array}{l}
P=\frac{1}{4 \pi} \int\left(u^{\prime} \frac{\partial \frac{1}{r}}{\partial x^{\prime}}+v^{\prime} \frac{\partial \frac{1}{r}}{\partial y^{\prime}}+w^{\prime} \frac{\partial \frac{1}{r}}{\partial z^{\prime}}\right) d t^{\prime}, \\
P_{23}=\frac{1}{4 \pi} \int\left(g^{\prime} \frac{\partial \frac{1}{r}}{\partial x^{\prime}}-w^{\prime} \frac{\partial \frac{1}{r}}{\partial y^{\prime}}+v^{\prime} \frac{\partial \frac{1}{r}}{\partial z^{\prime}}\right) d t^{\prime}, \\
P_{31}=\frac{1}{4 \pi} \int\left(w^{\prime} \frac{\partial \frac{1}{r}}{\partial x^{\prime}}+g^{\prime} \frac{\partial \frac{1}{r}}{\partial y^{\prime}}-u^{\prime} \frac{\partial \frac{1}{r}}{\partial z^{\prime}}\right) d t^{\prime}, \\
P_{12}=\frac{1}{4 \pi} \int\left(-v^{\prime} \frac{\partial \frac{1}{r}}{\partial x^{\prime}}+u^{\prime} \frac{\partial \frac{1}{r}}{\partial y^{\prime}}+g^{\prime} \frac{\partial \frac{1}{r}}{\partial z^{\prime}}\right) d t^{\prime}
\end{array}\right.
$$

Diese Functionen haben überdies die Eigenschaft, wenn $x, y, z$ die Coordinaten eines Punktes bezeichnen, der ausserhalb des Raumes $T$ liegt, in die Ausdrücke auf der rechten Seite der Gleichungen $\left(1^{b}\right.$.) eingesetzt, die Werthe Null zu produciren. Die angegebene Gestalt der Auflösung kann durch Anwendung einer theilweisen Integration, wie folgt, in eine andere verwandelt werden. Wenn das Element der Oberfläche $S$ in dem Punkte $\left(x^{\prime}, y^{\prime}, z^{\prime}\right)$ mit $d s^{\prime}$ be- 
zeichnet wird, wenn die nach $d s^{\prime}$ auszuführende Integration sich auf die ganze Oberfläche $S$ bezieht, wenn die in dem Punkte $\left(x^{\prime}, y^{\prime}, z^{\prime}\right)$ von dem Raume $T$ nach aussen gezogene Flächennormale $n^{\prime}$ mit den Axen der positiven $x, y, z$ die Winkel $\alpha^{\prime}, \beta^{\prime}, \gamma^{\prime}$ bildet, so hat man zunächst für einen Punkt $(x, y, z)$, der ausserhalb $T$ liegt, dann aber auch, wie leicht zu beweisen, für einen Punkt $(x, y, z)$, der innerhalb $\boldsymbol{T}$ liegt, die Gleichung

$$
\int u^{\prime} \frac{\partial \frac{1}{r}}{\partial x^{\prime}} d t^{\prime}=\int u^{\prime} \cos \alpha^{\prime} \frac{d s^{\prime}}{r}-\int \frac{\partial u^{\prime}}{\partial x^{\prime}} \frac{d t^{\prime}}{r} .
$$

Wir setzen hier voraus, dass die nach $x, y, z$ genommenen Differentialquotienten der Functionen $u, v, w, g$ überall in $T$ endliche Werthe haben. Werden die sämmtlichen Bestandtheile der für $\boldsymbol{P}, \boldsymbol{P}_{23}, \boldsymbol{P}_{31}, \boldsymbol{P}_{12}$ gefundenen Ausdrücke durch die entsprechenden Gleichungen umgeformt, und führt man die folgenden Bezeichnungen ein

$$
\left\{\begin{array}{l}
\boldsymbol{F}=-\frac{1}{4 \pi} \int\left(\frac{\partial u^{\prime}}{\partial x^{\prime}}+\frac{\partial v^{\prime}}{\partial y^{\prime}}+\frac{\partial w^{\prime}}{\partial z^{\prime}}\right) \frac{d t^{\prime}}{r} \\
F_{23}=-\frac{1}{4 \pi} \int\left(\frac{\partial g^{\prime}}{\partial x^{\prime}}-\frac{\partial w^{\prime}}{\partial y^{\prime}}+\frac{\partial v^{\prime}}{\partial z^{\prime}}\right) \frac{d t^{\prime}}{r} \\
F_{31}=-\frac{1}{4 \pi} \int\left(\frac{\partial w^{\prime}}{\partial x^{\prime}}+\frac{\partial g^{\prime}}{\partial y^{\prime}}-\frac{\partial u^{\prime}}{\partial z^{\prime}}\right) \frac{d t^{\prime}}{r} \\
F_{12}=-\frac{1}{4 \pi} \int\left(-\frac{\partial v^{\prime}}{\partial x^{\prime}}+\frac{\partial u^{\prime}}{\partial y^{\prime}}+\frac{\partial g^{\prime}}{\partial z^{\prime}}\right) \frac{d t^{\prime}}{r}
\end{array}\right.
$$

und ferner

$$
\left\{\begin{array}{l}
f=\frac{1}{4 \pi} \int\left(u^{\prime} \cos \alpha^{\prime}+v^{\prime} \cos \beta^{\prime}+w^{\prime} \cos \gamma^{\prime}\right) \frac{d s^{\prime}}{r} \\
f_{23}=\frac{1}{4 \pi} \int\left(g^{\prime} \cos \alpha^{\prime}-w^{\prime} \cos \beta^{\prime}+v^{\prime} \cos \gamma^{\prime}\right) \frac{d s^{\prime}}{r} \\
f_{31}=\frac{1}{4 \pi} \int\left(w^{\prime} \cos \alpha^{\prime}+g^{\prime} \cos \beta^{\prime}-u^{\prime} \cos \gamma^{\prime}\right) \frac{d s^{\prime}}{r} \\
f_{12}=\frac{1}{4 \pi} \int\left(-v^{\prime} \cos \alpha^{\prime}+u^{\prime} \cos \beta^{\prime}+g^{\prime} \cos \gamma^{\prime}\right) \frac{d s^{\prime}}{r}
\end{array}\right.
$$

so ergiebt sich diese zweite Gestalt der Auflösung

$$
\text { (7.) } \quad P=F+f, \quad P_{23}=F_{23}+f_{23}, \quad P_{31}=F_{31}+f_{31}, \quad P_{12}=F_{12}+f_{12} \text {. }
$$

Durch die Voraussetzung $g=0$ gehen $F, F_{23}, F_{31}, F_{12}$ in die von Roch angegebenen Raumintegrale über, und demnach sind $f, f_{23}, f_{31}, f_{12}$ die denselben als Ergänzung entsprechenden Oberflächenintegrale. 
114 Lipschitz, Beitrag zur Theorie der linearen partiellen Differentialgleichungen.

Um die abgeleiteten Resultate mit der von Herrn Helmholtz gegebenen Auflösung des specielleren Problems zu vergleichen, kann man den folgenden Weg einschlagen. Es mögen die Grössen $F, F_{23}, F_{31}, F_{12}$ aus (5.) in die Ausdrücke, die in $\left(1^{b}\right.$.) rechts von den Gleichheitszeichen stehen, beziehungsweise statt $P, P_{23}, P_{31}, P_{12}$ substituirt werden, so sind vier in $T$ überall endliche Functionen $\mathfrak{t}, \mathfrak{v}, \mathfrak{w}, \mathfrak{g}$ durch die folgenden Gleichungen vollständig bestimmt

$$
\text { (8.) }\left\{\begin{aligned}
u-\mathfrak{u}= & \frac{\partial \boldsymbol{F}}{\partial x}+\frac{\partial \boldsymbol{F}_{12}}{\partial y}-\frac{\partial \boldsymbol{F}_{31}}{\partial z} \\
\mathfrak{v}-\mathfrak{v}= & -\frac{\partial \boldsymbol{F}_{12}}{\partial x}+\frac{\partial \boldsymbol{F}}{\partial y}+\frac{\partial \boldsymbol{F}_{23}}{\partial z} \\
\boldsymbol{w}-\mathfrak{w}= & \frac{\partial \boldsymbol{F}_{31}}{\partial x}-\frac{\partial \boldsymbol{F}_{23}}{\partial y}+\frac{\partial \boldsymbol{F}}{\partial z} \\
g-\mathfrak{g}= & \frac{\partial \boldsymbol{F}_{23}}{\partial x}+\frac{\partial \boldsymbol{F}_{31}}{\partial y}+\frac{\partial \boldsymbol{F}_{12}}{\partial z}
\end{aligned}\right.
$$

Aus diesen Gleichungen fliessen die folgenden

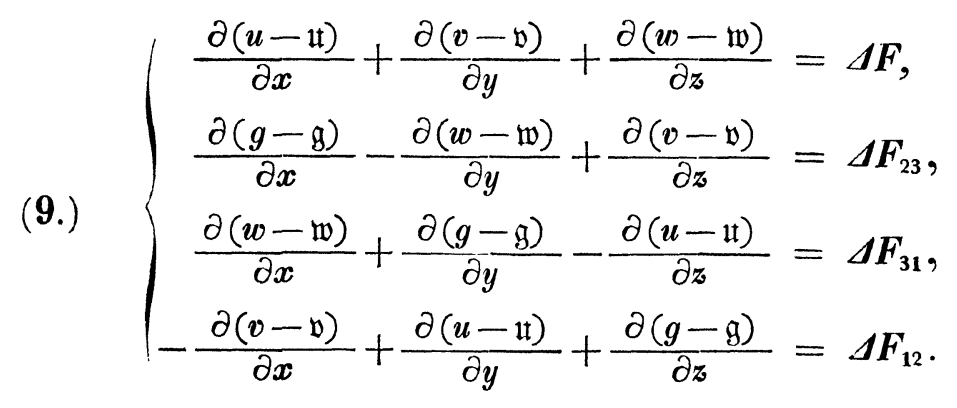

Dagegen ergeben sich aus (5.) die Relationen

$$
\text { (10.) }\left\{\begin{array}{l}
\frac{\partial u}{\partial x}+\frac{\partial v}{\partial y}+\frac{\partial w}{\partial z}=\Delta F \\
\frac{\partial g}{\partial x}-\frac{\partial w}{\partial y}+\frac{\partial v}{\partial z}=\Delta F_{23} \\
\frac{\partial w}{\partial x}+\frac{\partial g}{\partial y}-\frac{\partial u}{\partial z}=\Delta F_{31} \\
-\frac{\partial v}{\partial x}+\frac{\partial u}{\partial y}+\frac{\partial g}{\partial z}=\Delta F_{12}
\end{array}\right.
$$

und diese mit (9.) verbunden ziehen die folgenden Gleichungen zwischen den Functionen $\mathfrak{u}, \mathfrak{v}, \mathfrak{w}, \mathfrak{g}$ nach sich: 


$$
\left\{\begin{array}{r}
\frac{\partial \mathfrak{t}}{\partial x}+\frac{\partial \mathfrak{v}}{\partial y}+\frac{\partial \mathfrak{w}}{\partial \mathfrak{z}}=\mathbf{0} \\
\frac{\partial \mathfrak{g}}{\partial x}-\frac{\partial \mathfrak{w}}{\partial y}+\frac{\partial \mathfrak{v}}{\partial \mathfrak{z}}=\mathbf{0} \\
\frac{\partial \mathfrak{w}}{\partial x}+\frac{\partial \mathfrak{g}}{\partial y}-\frac{\partial \mathfrak{t}}{\partial \mathfrak{z}}=\mathbf{0} \\
-\frac{\partial \mathfrak{v}}{\partial x}+\frac{\partial \mathfrak{l}}{\partial y}+\frac{\partial \mathfrak{g}}{\partial \mathfrak{z}}=\mathbf{0}
\end{array}\right.
$$

Dieselben bieten in dem Falle, dass $\mathfrak{g}$ inı Innern von $\boldsymbol{T}$ überall gleich der Null ist, eine Handhabe, um eine Auflösung des Systems (1 ${ }^{b}$.) abzuleiten. Denn die drei letzten von den Gleichungen (11.) gehen alsdann in die nothwendigen und hinreichenden Bedingungen über, damit der Ausdruck $\mathfrak{l} d x+\mathfrak{v} d y+\mathfrak{w} d z$ ein vollständiges Differential sei. Beschreibt man also im Raume $T$ von einem beliebigen festen Punkte $\left(x_{0}, y_{0}, z_{0}\right)$ bis zu dem Punkte $(x, y, z)$ eine willkürliche Curve, so liefert eine längs derselben ausgeführte Integration die Function

$$
\text { (12.) } \mathfrak{B}=\int(\mathfrak{u} d x+\mathfrak{v} d y+\mathfrak{w} d \mathfrak{z}) \text {. }
$$

Die auf zwei verschiedenen von $\left(x_{0}, y_{0}, z_{0}\right)$ nach $(x, y, z)$ gezogenen Integrationswegen erhaltenen Werthe $\mathfrak{P}$ sind dieselben, wenn man eine Fläche construiren kann, welche nur diese beiden Integrationswege zur Begrenzung hat und ganz im Raume $T$ liegt; diese Werthe können aber verschiedene werden, wenn sich diese Bedingung nicht erfüllen lässt. Nunmehr bestehen die Gleichungen

$$
\text { (13.) } \quad \mathfrak{u}=\frac{\partial \mathfrak{P}}{\partial x}, \quad \mathfrak{v}=\frac{\partial \mathfrak{P}}{\partial y}, \quad \mathfrak{w}=\frac{\partial \mathfrak{B}}{\partial \mathfrak{z}},
$$

und wegen der ersten Gleichung (11.) gilt die Relation

$$
\text { (14.) } \mathscr{A P}=0 \text {. }
$$

Wenn jetzt die Werthe (13.) in (8.) substituirt werden, so ergiebt sich das System von Gleichungen

$$
\left\{\begin{array}{l}
\boldsymbol{u}=\frac{\partial(\boldsymbol{F}+\mathfrak{P})}{\partial x}+\frac{\partial \boldsymbol{F}_{12}}{\partial y}-\frac{\partial \boldsymbol{F}_{31}}{\partial z}, \\
\boldsymbol{v}=-\frac{\partial \boldsymbol{F}_{12}}{\partial \boldsymbol{x}}+\frac{\partial(\boldsymbol{F}+\mathfrak{P})}{\partial y}+\frac{\partial \boldsymbol{F}_{23}}{\partial \boldsymbol{z}}, \\
\boldsymbol{w}=\frac{\partial \boldsymbol{F}_{31}}{\partial \boldsymbol{x}}-\frac{\partial \boldsymbol{F}_{23}}{\partial y}+\frac{\partial(\boldsymbol{F}+\mathfrak{P})}{\partial z} \\
\boldsymbol{g}=\frac{\partial \boldsymbol{F}_{23}}{\partial x}+\frac{\partial \boldsymbol{F}_{31}}{\partial y}+\frac{\partial \boldsymbol{F}_{12}}{\partial z}
\end{array}\right.
$$


116 Lipschitz, Beitrag zur Theorie der linearen partiellen Differentialgleichungen.

welches lehrt, dass unter der Voraussetzung $\mathfrak{g}=0$ die Ausdrücke

(16.) $\quad P=F+\mathfrak{P}, \quad P_{23}=F_{23}, \quad P_{31}=F_{31}, \quad P_{12}=F_{12}$

eine Auflösung des Systems $\left(1^{b}\right.$.) darstellen. Ich werde nun die Grössen $\mathfrak{u}_{\text {, }}$ $\mathfrak{v}, \mathfrak{w}, \mathfrak{g}$ durch Oberflächenintegrale ausdrücken, um die Bedeutung der Gleichung $\mathfrak{g}=\mathbf{0}$ und das Wesen der Function $\mathfrak{B}$ in ein helleres Licht zu setzen. Aus (8.) ergiebt sich der folgende Werth von $\mathfrak{g}$

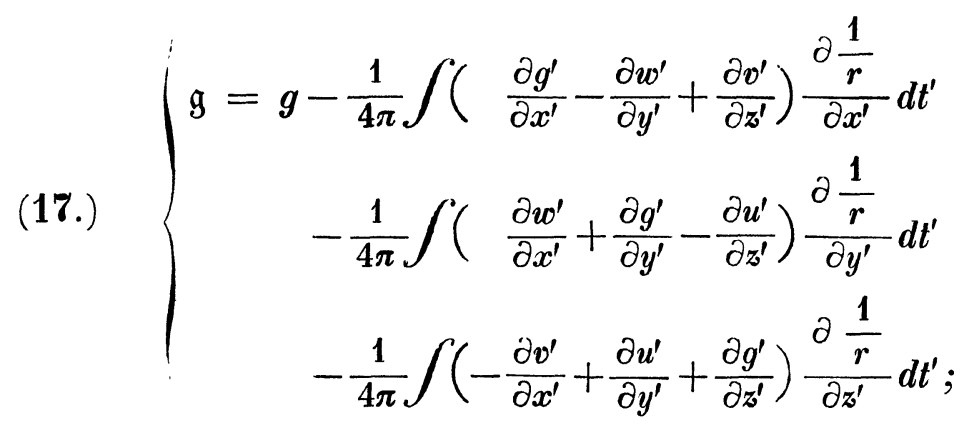

aus diesen bildet sich unter der Voraussetzung, dass die zweiten nach $x, y, z$ genommenen Differentialquotienten der Functionen $u, v, w, g$ in $\boldsymbol{T}$ überall endlich sind, mit Hülfe der oben angewendeten theilweisen Integration der Ausdruck

$$
\left\{\begin{aligned}
\mathfrak{g}=g & +\frac{1}{4 \pi} \int\left(\frac{\partial^{2} g^{\prime}}{\partial x^{\prime 2}}+\frac{\partial^{2} g^{\prime}}{\partial y^{\prime 2}}+\frac{\partial^{2} g^{\prime}}{\partial z^{\prime 2}}\right) \frac{d t^{\prime}}{r} \\
& -\frac{1}{4 \pi} \int\left(\frac{\partial g^{\prime}}{\partial x^{\prime}}-\frac{\partial w^{\prime}}{\partial y^{\prime}}+\frac{\partial v^{\prime}}{\partial z^{\prime}}\right) \cos \alpha^{\prime} \frac{d s^{\prime}}{r} \\
& -\frac{1}{4 \pi} \int\left(\frac{\partial w^{\prime}}{\partial x^{\prime}}+\frac{\partial g^{\prime}}{\partial y^{\prime}}-\frac{\partial u^{\prime}}{\partial z^{\prime}}\right) \cos \beta^{\prime} \frac{d s^{\prime}}{r} \\
& -\frac{1}{4 \pi} \int\left(-\frac{\partial v^{\prime}}{\partial x^{\prime}}+\frac{\partial u^{\prime}}{\partial y^{\prime}}+\frac{\partial g^{\prime}}{\partial z^{\prime}}\right) \cos \gamma^{\prime} \frac{d s^{\prime}}{r}
\end{aligned}\right.
$$

Da aber nach dem Greenschen Satze

$$
\text { (19.) }\left\{\begin{array}{l}
g+\frac{1}{4 \pi} \int\left(\frac{\partial^{2} g^{\prime}}{\partial x^{\prime 2}}+\frac{\partial^{2} g^{\prime}}{\partial y^{\prime 2}}+\frac{\partial^{2} g^{\prime}}{\partial z^{\prime 2}}\right) \frac{d t^{\prime}}{r} \\
=\frac{1}{4 \pi} \int\left(\frac{\partial g^{\prime}}{\partial x^{\prime}} \cos \alpha^{\prime}+\frac{\partial g^{\prime}}{\partial y^{\prime}} \cos \beta^{\prime}+\frac{\partial g^{\prime}}{\partial z^{\prime}} \cos \gamma^{\prime}\right) \frac{d s^{\prime}}{r} \\
-\frac{1}{4 \pi} \int g^{\prime}\left(\frac{\partial \frac{1}{r}}{\partial x^{\prime}} \cos \alpha^{\prime}+\frac{\partial \frac{1}{r}}{\partial y^{\prime}} \cos \beta^{\prime}+\frac{\partial \frac{1}{r}}{\partial z^{\prime}} \cos \gamma^{\prime}\right) d s^{\prime}
\end{array}\right.
$$

ist, so entsteht der gesuchte Ausdruck, wenn man der Kürze halber eine partielle Differentiation nach dem Element der Normale $\partial n^{\prime}$ einführt 


$$
\begin{gathered}
\left(20^{a} .\right) \quad \mathfrak{g}=-\frac{1}{4 \pi} \int g^{\prime} \frac{\partial \frac{1}{r}}{\partial n^{\prime}} d s^{\prime} \\
-\frac{1}{4 \pi} \int\left(\left(-\frac{\partial w^{\prime}}{\partial y^{\prime}}+\frac{\partial v^{\prime}}{\partial z^{\prime}}\right) \cos \alpha^{\prime}+\left(-\frac{\partial u^{\prime}}{\partial z^{\prime}}+\frac{\partial w^{\prime}}{\partial x^{\prime}}\right) \cos \beta^{\prime}+\left(-\frac{\partial v^{\prime}}{\partial x^{\prime}}+\frac{\partial u^{\prime}}{\partial y^{\prime}}\right) \cos \gamma^{\prime}\right) \frac{d s^{\prime}}{r} .
\end{gathered}
$$

Auch erhält man durch entsprechende Schritte die Ausdrücke

$$
\begin{aligned}
& \left\{\begin{aligned}
\mathfrak{u}=-\frac{1}{4 \pi} \int u^{\prime} \frac{\partial \frac{1}{r}}{\partial n^{\prime}} d s^{\prime} & -\frac{1}{4 \pi} \int\left(\left(\frac{\partial v^{\prime}}{\partial y^{\prime}}+\frac{\partial w^{\prime}}{\partial z^{\prime}}\right) \cos \alpha^{\prime}\right. \\
& \left.+\left(\frac{\partial g^{\prime}}{\partial z^{\prime}}-\frac{\partial v^{\prime}}{\partial x^{\prime}}\right) \cos \beta^{\prime}+\left(-\frac{\partial w^{\prime}}{\partial x^{\prime}}-\frac{\partial g^{\prime}}{\partial y^{\prime}}\right) \cos \gamma^{\prime}\right) \frac{d s^{\prime}}{r}
\end{aligned}\right. \\
& \left(20^{b} .\right)\left\{\mathfrak{v}=-\frac{1}{4 \pi} \int^{\partial} v^{\prime} \frac{\partial \frac{1}{r}}{\partial n^{\prime}} d s^{\prime}-\frac{1}{4 \pi} \int\left(\left(-\frac{\partial u^{\prime}}{\partial y^{\prime}}-\frac{\partial g^{\prime}}{\partial x^{\prime}}\right) \cos \alpha^{\prime}\right.\right. \\
& \left.+\left(\frac{\partial w^{\prime}}{\partial z^{\prime}}+\frac{\partial u^{\prime}}{\partial x^{\prime}}\right) \cos \beta^{\prime}+\left(\frac{\partial g^{\prime}}{\partial x^{\prime}}-\frac{\partial w^{\prime}}{\partial y^{\prime}}\right) \cos \gamma^{\prime}\right) \frac{d s^{\prime}}{r}, \\
& \mathfrak{w}=-\frac{1}{4 \pi} \int w^{\prime} \frac{\partial \frac{1}{r}}{\partial n^{\prime}} d s^{\prime}-\frac{1}{4 \pi} \int\left(\left(\frac{\partial g^{\prime}}{\partial y^{\prime}}-\frac{\partial u^{\prime}}{\partial z^{\prime}}\right) \cos \alpha^{\prime}\right. \\
& \left.+\left(-\frac{\partial v^{\prime}}{\partial z^{\prime}}-\frac{\partial g^{\prime}}{\partial x^{\prime}}\right) \cos \beta^{\prime}+\left(\frac{\partial u^{\prime}}{\partial x^{\prime}}+\frac{\partial v^{\prime}}{\partial y^{\prime}}\right) \cos \gamma^{\prime}\right) \frac{d s^{\prime}}{r} .
\end{aligned}
$$

Hier erscheint jede der Functionen $\mathfrak{t}, \mathfrak{v}, \mathfrak{w}, \mathfrak{g}$ als ein Aggregat von zwei Integralen, von denen das erste als das Potential einer magnetischen Doppelschicht auf der Oberfläche $S$, das zweite als das Potential einer magnetischen einfachen Schicht auf der Oberfläche $S$ in Bezug auf den Punkt $(x, y, z)$ betrachtet werden kann. Denkt man sich jetzt den Werth $g$ für die Oberfläche $S$ willkürlich gegeben, so ist in dem Ausdruck von $\mathfrak{g}$ das Potential der Doppelschicht vollständig bestimmt, und die Forderung, dass $\mathfrak{g}=0$ sei, führt nach einem Gaussischen Satze zu einer vollständigen Bestimmung der Dichtigkeit für die entsprechende einfache magnetische Schicht. Wird überdies angenommen, dass die Function $g$ an der ganzen Oberfläche $S$ den Werth Null habe, so muss die Dichtigkeit der einfachen Schicht überall ebenfalls gleich der Null sein, d. h. es muss für die ganze Oberfläche $\boldsymbol{S}$ die Gleichung gelten

$$
\mathbf{0}=\left(-\frac{\partial w}{\partial y}+\frac{\partial v}{\partial z}\right) \cos \alpha+\left(-\frac{\partial u}{\partial z}+\frac{\partial w}{\partial x}\right) \cos \beta+\left(-\frac{\partial v}{\partial x}+\frac{\partial u}{\partial y}\right) \cos \gamma
$$

Bei der von Herrn Helmholtz behandelten Aufgabe ist die Function $g$ in dem ganzen Raume $F$ gleich der Null, also drückt hier die vorstehende Gleichung 
118 Lipschitz, Beitrag zur Theorie der linearen partiellen Differentialgleichungen.

die zu dem Verschwinden der Grösse g nothwendige und hinreichende Bedingung aus. Unter der Voraussetzung $\mathfrak{g}=0$ bilden die Ausdrücke (16.) eine Auflösung des Systems $\left(1^{b}\right.$.), und dieselbe stimmt unter der ferneren Annahme, dass überall in $\boldsymbol{T}$ die Gleichung $\frac{\partial u}{\partial x}+\frac{\partial v}{\partial y}+\frac{\partial w}{\partial z}=0$ sei, mit der am angeführten Orte gegebenen Auflösung überein.

\section{3.}

Die Deutung der Functionen, die in den entwickelten Auflösungen unserer Aufgabe auftreten, erfordert, dass die Wirkung eines magnetischen Massenpunktes und eines electrischen Stromelements auf einen anderen magnetischen Massenpunkt vergleichbar dargestellt werden. In dem Punkte $(x, y, z)$, der die Wirkung erfährt, sei die Einheit des negativen (südlichen) magnetischen Fluidums concentrirt. Dann sind die Componenten der Wirkung, welche die im Punkte $\left(x^{\prime}, y^{\prime}, z^{\prime}\right)$ befindliche magnetische Masse $\iota^{\prime}$ ausübt, nach der $x, y, z$ Axe genommen, beziehungsweise

$$
\frac{\partial \frac{\mu^{\prime}}{r}}{\partial x}, \quad \frac{\partial \frac{\mu^{\prime}}{r}}{\partial y}, \quad \frac{\partial \frac{\mu^{\prime}}{r}}{\partial z} .
$$

Ferner sind die Componenten der Wirkung, welche das im Punkte $\left(x^{\prime}, y^{\prime}, z^{\prime}\right)$ befindliche mit der Intensität $\boldsymbol{J}^{\prime}$ durchströmte Linearelement $d l^{\prime}$ ausübt, nach der $x, y, z$ Axe genommen, wenn die positiven Axen der $x, y, z$ resp. nach oben, links, rechts gerichtet sind, beziehungsweise *)

$$
\begin{aligned}
& \frac{\partial \frac{J^{\prime}}{r} \frac{\partial y^{\prime}}{\partial l^{\prime}} d l^{\prime}}{\partial z}-\frac{\partial \frac{J^{\prime}}{r} \frac{\partial z^{\prime}}{\partial l^{\prime}} d l^{\prime}}{\partial y}, \\
& \frac{\partial \frac{\boldsymbol{J}^{\prime}}{r} \frac{\partial z^{\prime}}{\partial l^{\prime}} d l^{\prime}}{\partial \boldsymbol{x}}-\frac{\partial \frac{\boldsymbol{J}^{\prime}}{r} \frac{\partial x^{\prime}}{\partial l^{\prime}} d l^{\prime}}{\partial z}, \\
& \frac{\partial \frac{\boldsymbol{J}^{\prime}}{r} \frac{\partial x^{\prime}}{\partial l^{\prime}} d l^{\prime}}{\partial y}-\frac{\partial \frac{\boldsymbol{J}^{\prime}}{r} \frac{\partial y^{\prime}}{\partial l^{\prime}} d l^{\prime}}{\partial x} .
\end{aligned}
$$

Wie bei einer Vertheilung magnetischer Massen die über sämmtliche Massen ausgedehnte Summe $\Sigma \frac{\mu^{\prime}}{r}$ das Potential der Massenvertheilung heisst, so möge

*) Diese Formeln gelten nach der Ampèreschen Theorie nur für constante geschlossene Ströme, und die allgemeine Anwendung derselben gründet sich auf eine mathematische Fiction. 
der Complex der drei über sämmtliche Elemente einer Stromvertheilung genommenen Summen

$$
\Sigma \frac{J^{\prime} \frac{\partial x^{\prime}}{\partial l^{\prime}} d l^{\prime}}{r}, \quad \Sigma \frac{J^{\prime} \frac{\partial y^{\prime}}{\partial l^{\prime}} d l^{\prime}}{r}, \quad \Sigma \frac{J^{\prime} \frac{\partial z^{\prime}}{\partial l^{\prime}} d l^{\prime}}{r}
$$

das Potentialsystem der Stromvertheilung genannt werden. Wenn demnach bei einer Auflösung des Systems $\left(1^{b}\right.$.) die Function $P$ als das Potential einer Vertheilung magnetischer Massen, der Complex $-\boldsymbol{P}_{23},-\boldsymbol{P}_{31},-\boldsymbol{P}_{12}$ als das Potentialsystem einer Vertheilung von electrischen Strömen aufgefasst werden kann, so stellen die Functionen $u, v, w$ die Componenten der Wirkung dar, die von den magnetischen Massen und den electrischen Strömen gemeinsam auf den Punkt $(x, y, z)$ ausgeübt wird. Eine solche Auffassung der für $P, P_{23}$, $P_{31}, P_{12}$ gefundenen Ausdrücke kann nun folgendermassen vermittelt werden.

Es werde durch den Punkt $\left(x^{\prime}, y^{\prime}, z^{\prime}\right)$ oder $E$ ein System von drei auf einander senkrechten Linien gelegt, und es mögen auf jeder derselhen in je zwei gleichen Abständen von $\boldsymbol{E}$ zwei Punkte bestimmt werden, auf der ersten $\boldsymbol{E}_{\alpha}$ und $\boldsymbol{E}_{-\alpha}$, auf der zweiten $\boldsymbol{E}_{\beta}$ und $\boldsymbol{E}_{-\beta}$, auf der dritten $\boldsymbol{E}_{\gamma}$ und $\boldsymbol{E}_{-\gamma}$; die Linien seien von $E$ aus so gezogen, dass die Punkte $E_{\alpha}, E_{\beta}, E_{\gamma}$ beziehungsweise oben, links, rechts liegen. Die Punkte $E_{\alpha}, E_{\beta}, E_{\gamma}$ sollen in Bezug auf den Punkt $E$ die relativen Coordinaten $x=\alpha, y=\alpha_{1}, z=\alpha_{2}$; $x=\beta, y=\beta_{1}, z=\beta_{2} ; x=\gamma, y=\gamma_{1}, z=\gamma_{2}$ haben. Jetzt betrachte ich zuerst das folgende Gebilde. In dem Punkte $E_{\alpha}$ sei die magnetische Masse $\mu^{\prime}$, in dem Punkte $E_{-\alpha}$ die Masse $-\boldsymbol{\mu}^{\prime}$ concentrirt. In den Punkten $\boldsymbol{E}_{\beta}$ und $\boldsymbol{E}_{-\beta}$ werden parallele Linien zu der Linie $E_{-\gamma} E_{\gamma}$, in den Punkten $E_{\gamma}$ und $E_{-\gamma}$ parallele Linien zu der Linie $E_{-\beta} E_{\beta}$ gezogen, und das aus diesen vier sich schneidenden Linien gebildete Rechteck möge durch einen electrischen Strom von der Intensität $J^{\prime}$ in der Reihenfolge $E_{\beta}, E_{\gamma}, E_{-\beta}, E_{-\gamma}$ durchströmt werden. Ausserdem wird vorausgesetzt, dass die Länge der Linien $\boldsymbol{E}_{-\alpha} \boldsymbol{E}_{\alpha}, \boldsymbol{E}_{-\beta} \boldsymbol{E}_{\beta}$, $E_{-\gamma} E_{\gamma}$ gegen den Abstand des Punktes $E$ von dem Punkte $(x, y, z)$, der die anziehende Wirkung erfährt, kleine Grössen sind. Alsdann liefert das System der magnetischen Massen $\mu^{\prime}$ und $-\mu^{\prime}$, welches man einen Elementarmagneten nennen kann, das Potential

$$
\text { (21.) } 2 \mu^{\prime}\left(\frac{\partial \frac{1}{r}}{\partial x^{\prime}} \alpha+\frac{\partial \frac{1}{r}}{\partial y^{\prime}} \alpha_{1}+\frac{\partial \frac{1}{r}}{\partial z^{\prime}} \alpha_{2}\right) \text {. }
$$

Was das Potentialsystem des construirten geschlossenen Elementarstromes an- 
120 Lipschitz, Beitrag zur Theorie der linearen partiellen Differentialgleichungen.

langt, so liefern zu dem Ausdruck $\Sigma \frac{J \frac{\partial x^{\prime}}{\partial l^{\prime}} d l^{\prime}}{r}$ die Theile des Stromes, welche durch die Punkte $E_{\beta}$ und $E_{-\beta}$ hindurchgehen, den Beitrag

$$
4 \boldsymbol{J}^{\prime} \gamma\left(\frac{\partial \frac{1}{r}}{\partial x^{\prime}} \beta+\frac{\partial \frac{1}{r}}{\partial y^{\prime}} \beta_{1}+\frac{\partial \frac{1}{r}}{\partial z^{\prime}} \beta_{2}\right) \text {, }
$$

die Theile des Stromes, welche durch die Punkte $E_{\gamma}$ und $E_{-\gamma}$ hindurchgehen, den Beitrag

$$
-4 J^{\prime} \beta\left(\frac{\partial \frac{1}{r}}{\partial x^{\prime}} \gamma+\frac{\partial \frac{1}{r}}{\partial y^{\prime}} \gamma_{1}+\frac{\partial \frac{1}{r}}{\partial z^{\prime}} \gamma_{2}\right) \text {. }
$$

Wenn man nun die positiven Quadratwurzeln $\gamma\left(\alpha^{2}+\alpha_{1}^{2}+\alpha_{2}^{2}\right)=a, \jmath^{\prime}\left(\beta^{2}+\beta_{1}^{2}+\beta_{2}^{2}\right)$ $=\mathfrak{b}, \mathrm{l}^{\prime}\left(\gamma^{2}+\gamma_{1}^{2}+\gamma_{2}^{2}\right)=\mathrm{c}$ setzt, und sich der bekannten Formeln

$$
\begin{aligned}
& \beta_{1} \gamma_{2}-\beta_{2} \gamma_{1}=\frac{\mathfrak{b} \mathfrak{c}}{\mathfrak{a}} \alpha, \\
& \beta_{2} \gamma-\beta \gamma_{2}=\frac{\mathfrak{b} \mathfrak{c}}{\mathfrak{a}} \alpha_{1}, \\
& \beta \gamma_{1}-\beta_{1} \gamma=\frac{\mathfrak{b} \mathfrak{c}}{\mathfrak{a}} \alpha_{2}
\end{aligned}
$$

bedient, so nimmt das Potentialsystem des in Rede stehenden Stromrechtecks die Form an

(22.)

$$
\left\{\begin{array}{c}
-\frac{4 \mathfrak{b} c}{\mathfrak{a}} J^{\prime}\left(\frac{\partial \frac{1}{r}}{\partial y^{\prime}} \alpha_{2}-\frac{\partial \frac{1}{r}}{\partial z^{\prime}} \alpha_{1}\right) \\
-\frac{4 \mathfrak{b} \mathfrak{c}}{\mathfrak{a}} J^{\prime}\left(\frac{\partial \frac{1}{r}}{\partial z^{\prime}} \alpha-\frac{\partial \frac{1}{r}}{\partial x^{\prime}} \alpha_{2}\right) \\
-\frac{4 \mathfrak{b} \mathfrak{c}}{\mathfrak{a}} J^{\prime}\left(\frac{\partial \frac{1}{r}}{\partial x^{\prime}} \alpha_{1}-\frac{\partial \frac{1}{r}}{\partial y^{\prime}} \alpha\right)
\end{array}\right.
$$

Ich gehe nun zur Betrachtung eines zweiten Gebildes über. Es sei $\boldsymbol{E}_{\alpha}$ die Mitte eines Stromelements von der Länge $2 \mathfrak{a}$, bei welchem die Richtung des Stromes von dem Punkte $E$ nach dem Punkte $E_{\alpha}$ geht, ebenso $E_{-\alpha}$ die Mitte eines Stromelements von der Länge $2 \mathfrak{a}$, bei welchem die Richtung des Stromes die entgegengesetzte ist. In gleicher Weise seien $E_{\beta}$ und $E_{-\beta}$ die Mitten von Stromelementen mit der Länge $2 \mathfrak{a}$ und den Richtungen $E E_{\beta}, E E_{-\beta}$, und $E_{\gamma}$ und $E_{-\gamma}$ die Mitten von Stromelementen mit der Länge $2 \mathfrak{a}$ und den 
Richtungen $E E_{\gamma}, E E_{-\gamma}$. Die Stromstärke in allen Theilen dieses Stromkreuzes habe den Werth $\boldsymbol{J}^{\prime \prime}$. Dann wird das Potentialsystem des Stromkreuzes, wie leicht zu übersehen, das folgende
(23.)
$4 \mathfrak{a}^{2} J^{\prime \prime} \frac{\partial \frac{1}{r}}{\partial x^{\prime}}$,
$4 \mathfrak{a}^{2} J^{\prime \prime} \frac{\partial \frac{1}{r}}{\partial \dot{y}^{\prime}}$,
$4 \mathfrak{a}^{2} J^{\prime \prime} \frac{\partial \frac{1}{r}}{\partial z^{\prime}}$.

Wir machen jetzt die mathematische Fiction, dass die Wirkung sowohl der Elementarmagneten, als der Stromvierecke, als der Stromkreuze auch dann noch durch die angegebenen Ausdrücke dargestellt werde, wenn der in Angriff genommene Punkt $(x, y, z)$ in die Nähe jener Gebilde gerückt wird. Alsdann können wir eine Vertheilung von magnetischen Massen construiren, deren Potential gleich dem Werthe $P$ aus (4.) ist, und eine Vertheilung von electrischen Strömen angeben, deren Potentialsystem gleich dem Complex der Werthe $P_{23}, P_{31}, P_{12}$ aus (4.) ist. Man erhält nämlich das Element des für $P$ gefundenen Integrals, indem man in (21.) die Substitution macht

$$
\text { (24.) } \quad 2 \iota^{\prime} \alpha=\frac{1}{4 \pi} u^{\prime} d t^{\prime}, \quad 2 \iota^{\prime} \alpha_{1}=\frac{1}{4 \pi} v^{\prime} d t^{\prime}, \quad 2 \iota^{\prime} \alpha_{2}=w^{\prime} d t^{\prime},
$$

und man erhält die Elemente der für $P_{23}, P_{31}, P_{12}$ gefundenen Integrale, indem man in (22.) die Substitution

$$
\frac{4 \mathfrak{b} \mathfrak{c}}{\mathfrak{a}} J^{\prime} \alpha=\frac{1}{4 \pi} u^{\prime} d t^{\prime}, \quad \frac{4 \mathfrak{b} \mathfrak{c}}{\mathfrak{a}} J^{\prime} \alpha_{1}=\frac{v^{\prime}}{4 \pi} d t^{\prime}, \quad \frac{4 \mathfrak{b} \mathfrak{c}}{\mathfrak{a}} J^{\prime} \alpha_{2}=\frac{w^{\prime}}{4 \pi} d t^{\prime},
$$

und in (23.) die Substitution

$$
\text { (26.) } \quad 4 \mathfrak{a}^{2} J^{\prime \prime}=\frac{1}{4 \pi} g^{\prime} d t^{\prime}
$$

ausführt, dann aber die entsprechenden Ausdrücke addirt. Durch die Gleichungen (24.) wird die Richtung der Axe $E_{-\alpha} E_{\alpha}$ des Elementarmagneten und der Werth seines Moments $2 \mu^{\prime} \mathfrak{a}$ vollständig bestimmt. Die Gleichungen (25.) determiniren die Ebene des Stromvierecks senkrecht gegen die Axe des eben bestimmten Elementarmagneten, und liefern für das Product der Stromstärke in den Flächenraum des umströmten Rechtecks die Gleichung

$$
4 \mathfrak{b c} J^{\prime}=2 \mu^{\prime} \mathfrak{a},
$$

nach welcher dieses Product gleich dem Moment des in Rede stehenden Elementarmagneten ist. Also besteht zwischen dem Elementarmagneten und dem Stromviereck die Beziehung, dass ihre Wirkung auf einen entfernten Punkt dieselbe ist. Die Gleichung (26.) zeigt, dass die bei den Stromkreuzen zu wählende Lage der Linien $E E_{\alpha}, E E_{\beta}, E E_{\gamma}$ eine willkürliche ist, und dass die denselben beizulegende Stromstärke $J^{\prime \prime}$ der Function $g$ proportional wird, 
folglich mit der Function $g$ zugleich verschwindet. Wenn man daher in jedem Punkte $\left(x^{\prime}, y^{\prime}, z^{\prime}\right)$ des Innern von $T$ einen Elementarmagneten fingirt, der durch die Gleichungen (24.) bestimmt ist, dagegen ein Stromviereck und ein Stromkreuz annimmt, für welche beziehungsweise die Gleichungen gelten

$$
\begin{gathered}
\left(25^{\prime \prime} .\right) \quad \frac{4 \mathfrak{b} \mathfrak{c}}{\mathfrak{a}} J^{\prime} \alpha=-\frac{1}{4 \pi} u^{\prime} d t^{\prime}, \quad \frac{4 \mathfrak{b} \mathfrak{c}}{\mathfrak{a}} J^{\prime} \alpha_{1}=-\frac{v^{\prime}}{4 \pi} d t^{\prime}, \quad \frac{4 \mathfrak{b} \mathfrak{c}}{\mathfrak{a}} J^{\prime} \alpha_{2}=-\frac{w^{\prime}}{4 \pi} d t^{\prime}, \\
\left(26^{a} .\right) \quad 4 \mathfrak{a}^{2} J^{\prime \prime}=-\frac{1}{4 \pi} g^{\prime} d t^{\prime},
\end{gathered}
$$

so hat man ein System von Magneten, welches das Potential $\boldsymbol{P}$ producirt, und ein System von electrischen Strömen, welches das Potentialsystem $-\boldsymbol{P}_{23},-\boldsymbol{P}_{31}$, $-P_{12}$ erzeugt. Dieses System von Kraftquellen bringt nach dem oben Bemerkten auf einen im Innern von $T$ gelegenen Punkt $(x, y, z)$ eine Wirkung hervor, deren nach den $x, y, z$ Axen genommene Componenten respective gleich den gegebenen Werthen $u, v, w$ sind, übt dagegen auf einen ausserhalb $T$ befindlichen Punkt $(x, y, z)$ gar keine Wirkung aus.

Die in den Gleichungen (7.) enthaltene Auflösung unserer Aufgabe gestattet, die Functionen $\boldsymbol{F}$ und $f$ als Potentiale von Vertheilungen magnetischer Massen im Raume $T$ und in der Oberfläche $S$, die Complexe $F_{23}, F_{31}, F_{12}$ und $f_{23}, f_{31}, f_{12}$ als Potentialsysteme von Vertheilungen electrischer Ströme im Raume $T$ und in der Oberfläche $S$ aufzufassen. Es werde in dem Punkte $\left(x^{\prime}, y^{\prime}, z^{\prime}\right)$ eine magnetische Masse $\mu^{\prime}$, ein Stromelement $d l^{\prime}$ mit der Intensität $J^{\prime}$ und ein Stromelement $d l^{\prime \prime}$ mit der Intensität $J^{\prime \prime}$ fingirt, so hat man für diesen Zweck die folgenden Gleichungen anzuwenden: erstens im Raume $T$

$$
\left\{\begin{aligned}
\mu^{\prime} & =-\frac{1}{4 \pi}\left(\frac{\partial u^{\prime}}{\partial x^{\prime}}+\frac{\partial v^{\prime}}{\partial y^{\prime}}+\frac{\partial w^{\prime}}{\partial z^{\prime}}\right) d t^{\prime}, \\
J^{\prime} \frac{\partial x^{\prime}}{\partial l^{\prime}} d l^{\prime} & =-\frac{1}{4 \pi}\left(-\frac{\partial w^{\prime}}{\partial y^{\prime}}+\frac{\partial v^{\prime}}{\partial z^{\prime}}\right) d t^{\prime}, \\
J^{\prime} \frac{\partial y^{\prime}}{\partial l^{\prime}} d l^{\prime} & =-\frac{1}{4 \pi}\left(-\frac{\partial u^{\prime}}{\partial z^{\prime}}+\frac{\partial w^{\prime}}{\partial x^{\prime}}\right) d t^{\prime}, \\
J^{\prime} \frac{\partial z^{\prime}}{\partial l^{\prime}} d l^{\prime} & =-\frac{1}{4 \pi}\left(-\frac{\partial v^{\prime}}{\partial x^{\prime}}+\frac{\partial u^{\prime}}{\partial y^{\prime}}\right) d t^{\prime}, \\
J^{\prime \prime} \frac{\partial x^{\prime}}{\partial l^{\prime \prime}} d l^{\prime \prime} & =-\frac{1}{4 \pi} \frac{\partial g^{\prime}}{\partial x^{\prime}} d t^{\prime}, \\
J^{\prime \prime} \frac{\partial y^{\prime}}{\partial l^{\prime \prime}} d l^{\prime \prime} & =-\frac{1}{4 \pi} \frac{\partial g^{\prime}}{\partial y^{\prime}} d t^{\prime}, \\
J^{\prime \prime} \frac{\partial z^{\prime}}{\partial l^{\prime \prime}} d l^{\prime \prime} & =-\frac{1}{4 \pi} \frac{\partial g^{\prime}}{\partial z^{\prime}} d t^{\prime},
\end{aligned}\right.
$$


und zweitens in der Oberfläche $S$

$$
\left\{\begin{aligned}
u^{\prime} & =\frac{1}{4 \pi}\left(u^{\prime} \cos \alpha^{\prime}+v^{\prime} \cos \beta^{\prime}+w^{\prime} \cos \gamma^{\prime}\right) d s^{\prime} \\
J^{\prime} \frac{\partial x^{\prime}}{\partial l^{\prime}} d l^{\prime} & =\frac{1}{4 \pi}\left(-w^{\prime} \cos \beta^{\prime}+v^{\prime} \cos \gamma^{\prime}\right) d s^{\prime} \\
J^{\prime} \frac{\partial y^{\prime}}{\partial l^{\prime}} d l^{\prime} & =\frac{1}{4 \pi}\left(-u^{\prime} \cos \gamma^{\prime}+w^{\prime} \cos \alpha^{\prime}\right) d s^{\prime} \\
J^{\prime} \frac{\partial z^{\prime}}{\partial l^{\prime}} d l^{\prime} & =\frac{1}{4 \pi}\left(-v^{\prime} \cos \alpha^{\prime}+u^{\prime} \cos \beta^{\prime}\right) d s^{\prime} \\
J^{\prime \prime} \frac{\partial x^{\prime}}{\partial l^{\prime \prime}} d l^{\prime \prime} & =\frac{1}{4 \pi} g^{\prime} \cos \alpha^{\prime} d s^{\prime} \\
J^{\prime \prime} \frac{\partial y^{\prime}}{\partial l^{\prime \prime}} d l^{\prime \prime} & =\frac{1}{4 \pi} g^{\prime} \cos \beta^{\prime} d s^{\prime} \\
J^{\prime \prime} \frac{\partial z^{\prime}}{\partial l^{\prime \prime}} d l^{\prime \prime} & =\frac{1}{4 \pi} g^{\prime} \cos \gamma^{\prime} d s^{\prime}
\end{aligned}\right.
$$

In Bezug auf die letzten Formeln kann man die Bemerkung machen, dass die mit $d l^{\prime \prime}$ bezeichneten Stromelemente senkrecht gegen die Oberfläche $S$, die mit $d l^{\prime}$ bezeichneten Stromelemente aber tangential zu der Oberfläche $S$ gerichtet sind.

\section{4.}

Die Betrachtungen, die in den angeführten Abhandlungen unter der Voraussetzung, dass die Function $g$ gleich Null ist, und dass $u, v$, w die Componenten der Geschwindigkeit einer den Raum $\boldsymbol{T}$ continuirlich erfüllenden Materie im Punkte $(x, y, z)$ bedeuten, an die Interpretation der Integrale $F, F_{23}, F_{31}$, $F_{12}$ angeknüpft sind, und die sich auf die mechanische Bedeutung der Ausdrücke $\frac{\partial u}{\partial x}+\frac{\partial v}{\partial y}+\frac{\partial w}{\partial z}, \frac{\partial v}{\partial z}-\frac{\partial w}{\partial y}, \frac{\partial w}{\partial x}-\frac{\partial u}{\partial z}, \frac{\partial u}{\partial y}-\frac{\partial v}{\partial x}$ beziehen, veranlassen eine Frage nach den Bedingungen, unter denen die Elemente der Integrale $f, f_{23}, f_{31}, f_{12}$, das ist, die in der Oberfläche $S$ supponirten magnetischen Massen und electrischen Ströme, verschwinden. Die betreffende Antwort ist aus der Gleichung

$$
\begin{gathered}
(u \cos \alpha+v \cos \beta+w \cos \gamma)^{2}+(-w \cos \beta+v \cos \gamma)^{2}+(-u \cos \gamma+x \cos \alpha)^{2} \\
+(-v \cos \alpha+u \cos \beta)^{2}=u^{2}+v^{2}+w^{2}
\end{gathered}
$$

abzuleiten, und lautet dahin, dass zu diesem Ende die Werthe $u, v, w$ in jedem Punkte von $S$ gleich Null werden müssen. 
Wir wollen nun auch den entgegenslehenden Fall erwägen, wo unter der Voraussetzung, dass die Function $g$ gleich Null ist, die Elemente der Integrale $F, F_{23}, F_{31}, F_{12}$ verschwinden, das ist, wo in dem ganzen Raume $T$ die Gleichungen gelten

$$
\text { (29.) }\left\{\begin{array}{c}
\frac{\partial u}{\partial x}+\frac{\partial v}{\partial y}+\frac{\partial w}{\partial z}=0 \\
\frac{\partial v}{\partial z}-\frac{\partial w}{\partial y}=0, \quad \frac{\partial w}{\partial x}-\frac{\partial u}{\partial z}=0, \quad \frac{\partial u}{\partial y}-\frac{\partial v}{\partial x}=0 .
\end{array}\right.
$$

Dann sind $u, v, w$ die nach $x, y, z$ genommenen partiellen Differentialquotienten einer Function $Q$, die mittelst der Integration eines vollständigen Differentials durch die Gleichung

$$
\text { (30.) } Q=\int(u d x+v d y+w d z)
$$

bestimmt ist, und die der partiellen Differentialgleichung

$$
\text { (31.) } \Delta Q=0
$$

genügt. Das System $\left(1^{b}\right.$.) wird gleichzeitig in Folge der Gleichungen (7.) durch die Ausdrücke $P=f, P_{23}=f_{23}, P_{31}=f_{31}, P_{12}=f_{12}$ befriedigt. Es kann nun das besondere Sachverhältniss obwalten, dass in gewissen Theilen der Oberfläche $S$ die in (28.) angegebenen supponirten magnetischen Massen $\mu^{\prime}$ verschwinden und nur die supponirten electrischen Ströme bleiben, während in den übrigen Theilen der Oberfläche $S$ die angegebenen Intensitäten der electrischen Ströme $J^{\prime}$ verschwinden, und nur die magnetischen Massen $\mu^{\prime}$ bleiben. Die electrischen Ströme von den Intensitäten $J^{\prime \prime}$ fallen wegen der Gleichung $g=0$ von selbst fort. Die Componenten der Wirkung der in Rede stehenden Combination von magnetischen Massen und electrischen Strömen sind dann in Folge der oben ausgeführten Bemerkungen für einen im Innern von $T$ gelegenen Punkt $(x, y, z)$ beziehungsweise gleich den Grössen $u, v, w$, dagegen für einen ausserhalb $T$ gelegenen Punkt $(x, y, z)$ gleich Null. Damit in einem Theile $S_{1}$ von $S$ die magnetischen Massen $\iota^{\prime}$ verschwinden, muss für jeden Punkt von $S_{1}$ die Gleichung

$$
\text { (32.) } u \cos \alpha+v \cos \beta+w \cos \gamma=0
$$

erfüllt sein. Das heisst, diejenige Richtung, welche mit den Axen $x, y, z$ die Winkel bildet, deren cosinus $\frac{u}{\sqrt{\left(u^{2}+v^{2}+w^{2}\right)}}, \frac{v}{\sqrt{\left(u^{2}+v^{2}+w^{2}\right)}}, \frac{w}{\sqrt{\left(u^{2}+v^{2}+w^{2}\right)}}$ sind, muss tangential zu der Fläche $S_{1}$ sein. Wenn dagegen in einem Theile $S_{2}$ von $S$ die Intensitäten $J^{\prime}$ verschwinden sollen, so muss für jeden Punkt 
von $S_{2}$ die Proportion

$$
u: v: w=\cos \alpha: \cos \beta: \cos \gamma
$$

befriedigt sein. Weil nun $u=\frac{\partial Q}{\partial x}, v=\frac{\partial Q}{\partial y}, w=\frac{\partial Q}{\partial z}$ ist, so zieht die Differentialgleichung der Fläche $\cos \alpha d x+\cos \beta d y+\cos \gamma d z=0$ die Gleichung $d Q=0$ nach sich. Daher muss in den zusammenhängenden Theilen $S_{2}$ von $S$ die Function $Q$, welche wegen (31.) eine Potentialfunction ist, einen constanten Werth haben, oder diese Theile müssen mit Niveauflächen der Potentialfunction $Q$ übereinstimmen.

Denkt man sich eine Potentialfunction $Q$ gegeben, so kann ein Raum $T$, dessen Oberfläche aus Theilen von der für $S_{1}$ und $S_{2}$ charakteristischen Beschaffenheit besteht, wie folgt gefunden werden. Zunächst sind die Linien aufzusuchen, welche auf den Niveauflächen von $Q$ überall senkrecht stehen. Wenn $(x, y, z)$ ein Punkt einer solchen Linie ist, so haben seine Coordinaten das System von Differentialgleichungen zu erfüllen

$$
\text { (34.) } \quad d x: d y: d z=\frac{\partial Q}{\partial x}: \frac{\partial Q}{\partial y}: \frac{\partial Q}{\partial z},
$$

und eine Linie, die durch einen gegebenen Punkt hindurchgehen muss, ist vollständig bestimmt. Jedes Integral $\boldsymbol{R}=$ const. dieses Systems von Differentialgleichungen genügt der unter dem Namen Charakteristik bekannten partiellen Differentialgleichung

$$
\text { (35.) } \frac{\partial \boldsymbol{Q}}{\partial x} \frac{\partial \boldsymbol{R}}{\partial x}+\frac{\partial \boldsymbol{Q}}{\partial y} \frac{\partial \boldsymbol{R}}{\partial y}+\frac{\partial \boldsymbol{Q}}{\partial z} \frac{\partial \boldsymbol{R}}{\partial z}=\mathbf{0},
$$

und die Theorie des Jacobischen Multiplicators lehrt, dass aus einem gegebenen Integral derselben das zur vollständigen Integration des Systems (34.) erforderliche zweite Integral durch die Integration eines vollständigen Differentials abgeleitet werden kann. Es wird angenommen, dass zwei independente Integrale von (35.) vorliegen. Wir denken uns nun in einer Niveaufläche $Q=A$ eine geschlossene Curve gezogen, in jedem Punkt dieser Curve die so eben bestimmte Linie construirt, und diese sämmtlichen Linien auf einer Seite der Fläche $Q=A$ von dieser bis zu einer zweiten Niveaufläche $Q=B$ genommen. Die Totatität der construirten Linien bildet dann eine Fläche, deren Gleichung auf die Form $\boldsymbol{\Phi}=$ const. gebracht werden kann, wo dle Function $\boldsymbol{\Phi}$, für $\boldsymbol{R}$ gesetzt, der partiellen Differentialgleichung (35.) genügt, mithin eine Function der beiden independenten Integrale ist. Wenn jetzt der von den Flächen $Q=A$, 
$Q=B, \Phi=$ const. eingeschlossene Raum so gewählt ist, dass innerhalb desselben die Functionen $Q, \frac{\partial Q}{\partial x}, \frac{\partial Q}{\partial y}, \frac{\partial Q}{\partial z}$ überall endlich bleiben, so leuchtet ein, dass, weil die Fläche $\Phi=$ const. den der Fläche $S_{1}$ vorgeschriebenen Charakter hat, und die Flächen $Q=A$ und $Q=B$ die den Flächenstücken $S_{2}$ vorgeschriebenen Eigenschaften besitzen, der in Rede stehende Raum die an den Raum $\boldsymbol{T}$ gestellten Anforderungen erfüllt.

Aus den Gleichungen (28.) geht hervor, dass die Dichtigkeit der in jedem Punkte der Flächen $Q=A$ und $Q=B$ zu supponirenden magnetischen Belegung erhalten wird, indem man den nach der äusseren Normale genommenen Differentialquotienten der Potentialfunction $Q$ durch $4 \pi$ dividirt. Um die Vertheilung der electrischen Ströme in der Fläche $\Phi=C$ darzustellen, fügen wir zu dieser Funclion $\Phi$ ein zweites von derselben unabhängiges Integral $\Phi_{1}$ der Differentialgleichung (35.) hinzu und drücken die Coordinaten eines Punktes der Fläche $\Phi=C$ durch die Functionen $Q$ und $\Phi_{1}$ aus. Dann giebt die Gleichung $Q=$ const. lauter in sich zurücklaufende Linien, und die Gleichung $\Phi_{1}=$ const. solche Linien, die auf den ersteren überall senkrecht stehen, und für die das System (34.) befriedigt ist. Bezeichnet man die Functionaldeterminante der Functionen $Q, \Phi, \Phi_{1}$ nach den Variabeln $x, y, z$ genommen, welche unter den bestehenden Verhältnissen nicht verschwinden kann, mit $D$, so hat das Element der Oberfläche $\Phi=C$ dell Ausdruck $\frac{\sqrt{\left(\left(\frac{\partial \Phi}{\partial x}\right)^{2}+\left(\frac{\partial \boldsymbol{\Phi}}{\partial y}\right)^{2}+\left(\frac{\partial \boldsymbol{\Phi}}{\partial z}\right)^{2}\right)}}{D}$ $d Q d \Phi_{1}$, und die betreffenden Formeln (28.) gehen, wenn man die Accente fortlässt, durch eine bekannte Transformation in die folgende Gestalt über

$$
\text { (36.) }\left\{\begin{array}{l}
J \frac{\partial x}{\partial l} d l=\frac{1}{4 \pi} \frac{\partial x}{\partial \Phi_{1}} d Q d \Phi_{1} \\
J \frac{\partial y}{\partial l} d l=\frac{1}{4 \pi} \frac{\partial y}{\partial \Phi_{1}} d Q d \Phi_{1} \\
J \frac{\partial z}{\partial l} d l=\frac{1}{4 \pi} \frac{\partial z}{\partial \Phi_{1}} d Q d \Phi_{1}
\end{array}\right.
$$

Demnach wird durch je zwei Niveauflächen, in denen der constante Werth des Potentials $Q$ um $d Q$ verschieden ist, aus der Fläche $\Phi=C$ ein Elementarstreifen herausgeschnitten, in welchem ein electrischer Strom von der Stärke $J=\frac{d Q}{4 \pi}$ zu fingiren ist. 
Für die Potentialfunction $Q=x$ werden die Niveauflächen $Q=$ const. mit der Ebene der $y-z$ parallele Ebenen, die Flächen $\Phi=$ const. auf der Ebene der $y-z$ senkrecht stehende Cylinderflächen. Alsdann geht das gefundene Resultat in den von Neumann Bd. 37 dieses Journals pag. 47 angeführten Satz über, nach welchem die Wirkung einer von constanten Strömen durchströmten Drahtspirale durch die Wirkung einer magnetischen Belegung ihrer beiden Grundflächen ersetzt werden kann.

Bonn, den 24. Februar 1868. 